\title{
Analysis of gamma index using in-house developed heterogeneous thorax phantom
}

\begin{abstract}
Quality assurance (QA) before patient treatment plays a major role in complex radiotherapy (RT) treatment planning. Phantoms are the standard materials that are used for the pretreatment QA of patients. Contemporary phantoms used in RT are mainly water-based, having simple geometry and of uniform density. These phantoms dose not replicate the exact heterogeneities of the actual human body. The present work aims to evaluate the results of relative dosimetry performed on in-house developed heterogeneous thorax phantom (HTP) using an electronic portal imaging device (EPID). To perform the relative dosimetry, contours were drawn on HTP at three different locations and intensity modulated radiotherapy (IMRT), as well as Rapid ARC (RA), were generated on it to compare the fluence obtained on TPS as well as on linac machine. The gamma evaluation results indicate a strong correlation between planned and the measured fluence. We found the EPID-based QA done on HTP explore its property as a QA tool and it can further be used to perform patient-specific relative dosimetry.
\end{abstract}

Keywords: electronic portal imaging device, heterogeneous thorax phantom, quality assurance

\author{
Volume 8 Issue I - 202 I
}

\author{
Priyusha Bagdare,' Swati Dubey, ${ }^{2}$ Sanjay \\ Kumar Ghosh, ${ }^{3}$ Shital Bhandigare ${ }^{4}$ \\ 'Research Scholar, School of Studies in Physics, Vikram \\ University, India \\ ${ }^{2}$ Associate Professor, School of Studies in Physics, Vikram \\ University, India \\ ${ }^{3}$ Professor \& Head, School of Studies in Physics, Vikram \\ University, India \\ ${ }^{4}$ D.Y. Patil Education Society (Institution Deemed to be \\ University), Kolhapur
}

Correspondence: Priyusha Bhaskar Bagdare, Research Scholar,
School of Studies in Physics, Vikram University, Ujjain (M.P.) 45600 I,Tel +91-8982 142093, Email priyushabagdar@gmail.com

Received: December 29, 2020 | Published: February 12, 202 |
Abbreviations: QA, quality assurance; RT, radiotherapy; HTP, heterogeneous thorax phantom; EPID, electronic portal imaging device; PTV, planning target volume; TPS, treatment planning system; IMRT, intensity-modulated radiotherapy; SSD, surface distance; DTA, distance-to-agreement; FFF, flattening filter-free; HD_MLC, high definition multi-leaf collimator; DD, dose-difference; PTV, planning target volume; TPS, treatment planning system; EPID, electronic portal imaging devices

\section{Introduction}

The high-end radiotherapy (RT) treatment technique needs an accurate pre-treatment patient quality assurance (QA) before patient treatment. ${ }^{1,2}$ In order to evaluate any RT plan or a treatment procedure two important aspects must be taken into account, first, an exact environment that can simulate radiation interaction as that of the real body tissue and second an accurate pre-treatment plan verification system. Phantoms are those substitutes that stimulate the real body scenario and have been in use since the inspection of RT. $3^{3-5}$ Although considering that the majority of the human body is consists of water, physical phantoms that are made of water or solid water equivalent materials are mostly used for the patient-specific QA.6-8 These phantoms are mostly used because of their cost-effectiveness and simpler designs. In contrast, knowing that apart from water, the human body is consists of bones, soft-tissues, air cavities, etc. of varied densities, anthropomorphic phantoms now a day used for the more pragmatic and finer representation of the human body. ${ }^{9-10}$ Regarding the patient plan verification, absolute point dosimetry and relative dosimetry are the two techniques mostly used in RT. To perform absolute dosimetry, an ion chamber is used inside the homogenous phantom to measure the point dose at a given point, and the same is verified on the machine by replicating the similar set-up geometry. However, to perform the relative dosimetry, various dosimetric devices are being used such as films, imatrix, ion chamber arrays, or flatpanel electronic portal imaging devices (EPID). In relative dosimetry two-dimensional fluence calculated by TPS is verified with that of the two-dimensional fluence obtained and on the linac. Amongst the all available equipment, EPIDs are the better option available to take real-time measurement along with good image resolution. ${ }^{11}$

The present work aims to explore the QA properties of in-house developed heterogeneous thorax phantom (HTP) which represent the actual thoracic region of the human body.

\section{Materials and Methods}

To design the HTP average dimension of thorax regions of 40 adult patients was taken irrespective of their gender and age distribution. Materials used for the designing of HTP are chosen such that it replicates the radiological characteristics of tissues involved, which has been described earlier in detail. ${ }^{12}$ Briefly, this phantom is made up of porous sawdust of pinewood (density: $0.24 \mathrm{~g} / \mathrm{cm} 3$ ) to mimic the lung, honeybee's wax of density (density: $0.86 \mathrm{~g} / \mathrm{cm} 3$ ) to replicate the soft-tissue region, and rib cage (density: $1.84 \mathrm{~g} / \mathrm{cm} 3$ ) to represent the actual human thoracic bony cavity as shown in Figure 1.

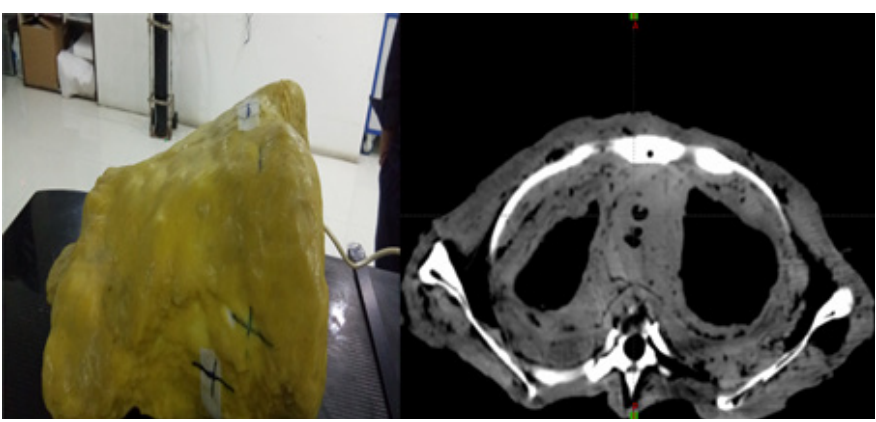

Figure I In-house developed heterogeneous thorax phantom.

CT images of the designed HTP with $3.0 \mathrm{~mm}$ slice thickness were taken on Siemens Biograph PETCT (Siemens Medical Systems, Germany). The corresponding CT images are then imported to the treatment planning system (TPS) eclipse version 13.7 (Varian Medical Systems, Palo Alto, CA, USA) to perform the relative dosimetry. 
To verify the property of HTP as a QA tool, planning target volume (PTV) was drawn on HTP at three different locations; i) on soft-tissue region ii) at the boundary of lung and bone and iii) inside the lung region as shown in figure 2 .

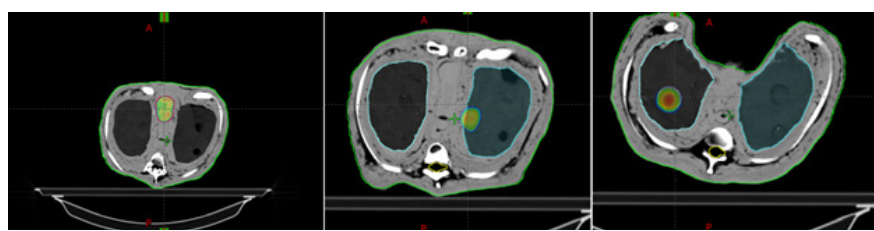

Figure 2 Target representation at three different locations inside the HTP.

Organs at risk (OARs) were also drawn along with the PTVs to evaluate the dose received by normal organs during RT. For each target, a plan was generated on a treatment planning system (TPS) using intensity-modulated radiotherapy (IMRT) as well as Rapid ARC (RA) technique and Acuros XB (AXB) version13.6.23 was used for dose calculation. The 2D-fluence generated by TPS on EPID (precalibrated aS1200-Varian Medical Systems, Palo Alto, CA) was exported to the machine for further analysis. Using the same setup as that was used in TPS; exported plans were exposed on Edge linac

Table I Results of all planning target volume locations

\begin{tabular}{|c|c|c|c|c|}
\hline PTV Location & Technique & Area Gamma & Maximum Dose Difference & Average Dose Difference \\
\hline Soft-tissue & RA & $100 \%$ & $11.1 \%$ & $1.2 \%$ \\
\hline Boundary of lung \& bone & RA & 99.9 & 13.7 & $1.6 \%$ \\
\hline Inside the lung & RA & $100 \%$ & $19.7 \%$ & $2.7 \%$ \\
\hline Soft-tissue & IMRT & $100 \%$ & $18.2 \%$ & $1.7 \%$ \\
\hline Boundary of lung \& bone & IMRT & $100 \%$ & $25.3 \%$ & $2.3 \%$ \\
\hline Inside the lung & IMRT & $100 \%$ & $23.8 \%$ & $3.2 \%$ \\
\hline
\end{tabular}

From the above table, it is clear that for all PTVs inside the HTP, the results are well within the tolerance limits and the HTP can be used as a QA tool for relative dosimetric purposes. Figure 3 represent the result of gamma value analysis on EPID for one of the RA plan in the soft-tissue region.

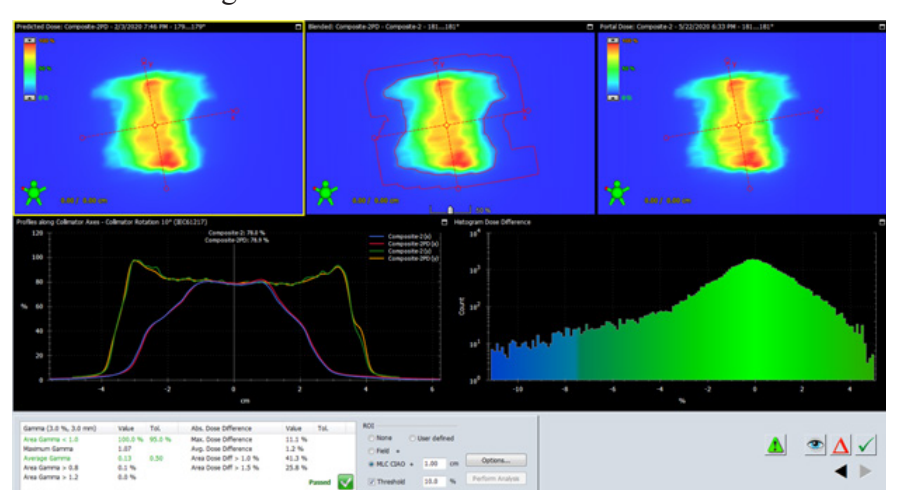

Figure 3 Gamma value analysis result for VMAT plan in soft-tissue region.

\section{Discussion}

The results obtained from relative dosimetry through HTP, which was based on the gamma-evaluation method showed that the fluence obtained on TPS and that obtained from the machine for all generated QA plans were matching as per the $3 \mathrm{~mm}, 3 \%$ gamma criteria Gamma index evaluations showed that no more than $3 \%$ of the points
(Varian Medical Systems, Palo Alto, CA) equipped with flattening filter-free (FFF) beams for facilitating higher dose rate, high definition multi-leaf collimator (HD-MLC) $(0.25 \mathrm{~cm})$ along with jaw tracking technology.

All measurements were taken on the EPID detector calibrated for source to the surface distance of (SSD) $100 \mathrm{~cm}$. The data were taken for the same gantry and collimator position which was there actually in the plan. 2D fluence obtained by TPS and that on the machine was compared and analyzed using Omni pro software (IBA Dosimetry, Germany). Pixel-based passing criteria were taken for plan evaluation, $g$ \# 1 was considered as pass criteria whereas $g>$ 1was taken as criteria for plan failure. For the acceptance criteria, we chose tolerance: 1) dose-difference (DD) of 3\% and distance-toagreement (DTA) of $3 \mathrm{~mm}$.

\section{Results}

In the previous study done by the author, ${ }^{11}$ physical and radiological properties of HTP were studied and compared with that of the actual patient. In the present study, the utility and accuracy of designed HTP as a QA tool is studied. The results obtained from relative dosimetry are shown in Table 1. within the range of $3 \mathrm{~mm}$ had a gamma value larger than one. ${ }^{12}$ The percentage of points satisfying the above passing criteria was found by comparing the TPS generated 2D gamma fluence with that of the fluence obtained on the machine.

\section{Conclusion}

The result of the current study indicates that EPID based relative dosimetry done on the HTP explores its property as a QA tool. Though in this study the contour was drawn on the HTP and the relative dosimetry was performed for the plans generated on it. But the further study can be carried out to perform patient-specific exit dosimetry on designed HTP to verify the patient's plans.

\section{References}

1. Svenesson GK. Quality assurance in radiation therapy: physical effort. Int J Radiat Oncol Biol Phys. 1984;10(1):23-29.

2. Brahme A, Chavaudra J, Landberg T, et al. Accuracy requirements and quality assurance of external beam therapy with photons and electrons. Acta Oncol. 1988;1(1):1-76.

3. Manikandan A, Sekaran SC, Sarkar B, et al. A homogeneous waterequivalent anthropomorphic phantom for dosimetric verification of radiotherapy plans. J Med Phys. 2018;43(2):100-105.

4. Letourneau D, Publicover J, Kozelka J, et al. Novel dosimetric phantom for quality assurance of volumetric modulated arc therapy. Med Phys. 2009;36(5):1813-1821. 
5. Gurjar OP, Mishra SP. A comparative study on patient-specific absolute dosimetry using slab phantom, acrylic body phantom, and goat head phantom. Int J Cancer Ther Oncol. 2015;3(2):1-5.

6. Hill R, Kuncic Z, Baldock C. The water equivalence of solid phantoms for low energy photon beams. Med Phys. 2010;37(8):4355-4363.

7. Shaiju VS, Kumar R, Phani D, et al. Design, fabrication, and validation of a polymethyl methacrylate head phantom for dosimetric verification of cranial radiotherapy treatment plans. J Med Phys. 2020;45(2):66-70.

8. Aslam A, Kakakhel MB, Shahid SA, et al. Soft tissue and water substitutes for megavoltage photon beams: An EGSnrc-based evaluation. J Appl Clin Med Phys. 2016;17(1):408-415.

9. Yasui K, Toshito T, Omachi C, et al. Dosimetric verification of IMPT using a commercial heterogeneous phantom. J Appl Clin Med Phys. 2019;20(2):114-120.
10. Gurjar OP, Mishra SP, Bhandari V, et al. Radiation dose verification using real tissue phantom in modern radiotherapy techniques. $\mathrm{J} \mathrm{Med}$ Phys. 2014;39(1):44-49.

11. Chen MW, Deng XW, Huang SM, et al. Application of amorphous silicon electronic portal imaging device (a-Si EPID) to dosimetry quality assurance of radiation therapy. Ai Zheng. 2007;26 (11):1272-1275.

12. Dubey S, Ghosh S, Bagdare P. Radiological properties of high energy $\mathrm{X}$-rays inside the in house developed heterogeneous thorax phantom. AIP Conference Proceedings 2224, 070002. International conference on Emerging Interfaces of Plasma Science and Technology, (EIPT-2019) AIP Conf Proc. 2020. 